\title{
Understanding Statistics 4
}

\author{
Elliot Abt \\ Department of Dentistry, Illinois Masonic Medical Center, Chicago, Illinois, USA
}

If the reader of an article can identify the type of data used in a study, this will be instrumental in determining the nature of the statistical analysis. As mentioned in the first article in this series, we have defined three domains of statistics: differences between groups, associations between groups and time-to-event data. This article will focus on statistics associated with the first domain: differences between groups.

\section{Categorical Data}

We will begin by examining eye colour, a type of categorical data. In a group of 100 boys, let's say 25 of them had blue eyes. If we had a group of 200 girls, assuming the proportions were identical, we would expect 50 of the girls, or $25 \%$ of them, to also have blue eyes. Statistics with categorical and its subset of binary data are about dealing with differences in proportions, and the chi-square test is a hypothesis test which provides a P-value, and works by evaluating these differences between groups. In this example, because the proportion $25 / 100$ is equivalent to $50 / 200$, the chi-square value would equal zero, which is the null value. As the difference in this proportion begins to change, the chi-square statistic will increase, and the corresponding P-value will begin to decrease. At some point, these differences may become large enough to reach statistical significance.

Binary data is a subset of categorical data and there are several statistical tests associated with this data type that readers of the literature are likely to encounter. These tests are risk ratios (RR), odds ratios (OR), absolute risk reduction (ARR) and number needed-totreat (NNT). To understand these principles, one must understand risk and odds.

Example: If four people are ice skating, and one person falls, we would say the risk of falling is simply the number who have experienced the event divided by the total number of people at risk. Thus the risk of the event is $1 / 4$, or $25 \%$, or 0.25 . However, the odds of

\begin{tabular}{l|c|c|c} 
Table 1 & \multicolumn{5}{|l}{} \\
\hline Exposure & Failure & Survival & Total \\
\hline Experimental implant & 2 & 8 & 10 \\
\hline Control implant & 6 & 4 & 10 \\
\hline Total & 8 & 12 & 20
\end{tabular}

\begin{tabular}{|c|c|c|c|}
\hline $\begin{array}{l}\text { Scaling Only pocket } \\
\text { depth }(\mathrm{mm})\end{array}$ & $\begin{array}{c}\text { Scaling }+ \text { Ab pocket } \\
\text { depth }(\mathrm{mm})\end{array}$ & Scaling Only Rank & Scaling + Ab Rank \\
\hline 5.0 & 4.6 & 4 & 1 \\
\hline 6.9 & 4.8 & 9 & 3 \\
\hline 6.0 & 5.2 & 8 & 6 \\
\hline 5.1 & 5.7 & 5 & 7 \\
\hline 7.0 & 4.7 & 10 & 2 \\
\hline
\end{tabular}

\begin{tabular}{c|c|c|c|}
\hline $\begin{array}{c}\text { Table } 3 \\
\text { Participant } \\
\text { (scaling group) }\end{array}$ & $\begin{array}{c}\text { Scaling Alone } \\
\text { (in mm) }\end{array}$ & $\begin{array}{c}\text { Scaling + Ab } \\
\text { (in mm) }\end{array}$ & $\begin{array}{c}\text { Participant (scaling } \\
\text { + Ab group) }\end{array}$ \\
\hline 1 & 5.0 & 4.6 & 6 \\
\hline 2 & 6.9 & 4.8 & 7 \\
\hline 3 & 6.0 & 5.2 & 8 \\
\hline 4 & 5.1 & 5.7 & 9 \\
\hline 5 & 7.0 & 4.7 & Mean \\
\hline
\end{tabular}

the event are calculated as the number experiencing the event divided by the number who have not experienced the event. Thus, the odds would be $1 / 3$, or 0.33 . Normally, odds are not expressed as a percentage.

Now let's look at Table 1 , often given in a $2 \times 2$ table, where we have two groups of patients, one with a control and one with an experimental dental implant. It appears that the experimental dental implant reduces the likelihood of failure, but we would like to quantify this difference.

The risk ratio (RR) is the incidence in the exposed group / incidence in non-exposed.

In this case the RR is incidence in experimental group / incidence in control group.
Thus the $\mathrm{RR}=2 / 10$

$$
6 / 10 \text { or } 0.33 \text {. }
$$

If both implant types had the same effect, both the RR and OR would take a value of 1 , which is the null value.

If the $R R<1$, experimental implant appears to decrease the failure rate

If the $R R>1$, experimental implant appears to increase the failure rate

Be careful if the outcome is survival, in which a beneficial result from the experimental implant would have a RR $>1$ and an adverse effect would have a $\mathrm{RR}<1$.

As the RR is 0.33 we would say that the risk of failure with the experimental implant 
is $1 / 3$ of what it is with the control implant, or that the risk of failure is reduced by $67 \%$.

The odds ratio (OR) is the odds in the exposed / odds in the non-exposed. Therefore the OR is $\underline{2 / 8}$

$$
6 / 4 \text { or } 0.17 \text {. }
$$

This means the odds of failure are $17 \%$ of what they are with control implant, or the odds of failure are reduced by $83 \%$ with the experimental implant.

ORs tend to exaggerate treatment effects, as the OR is always further away from the null value as compared with the RR ( 0.33 vs 0.17 ).

We can also calculate the ARR and NNT from this data set.

The ARR is simply the risk difference: $60 \%$ $-20 \%=40 \%$ meaning the risk of failure with the experimental implant is reduced by $40 \%$. The NNT is the reciprocal of the ARR (not expressed as a percentage) or $1 / 0.40=2.5$ (rounded to 3). This means we would have to treat 3 patients for the next patient to benefit from the experimental implant. NNTs are a way for practitioners to estimate the

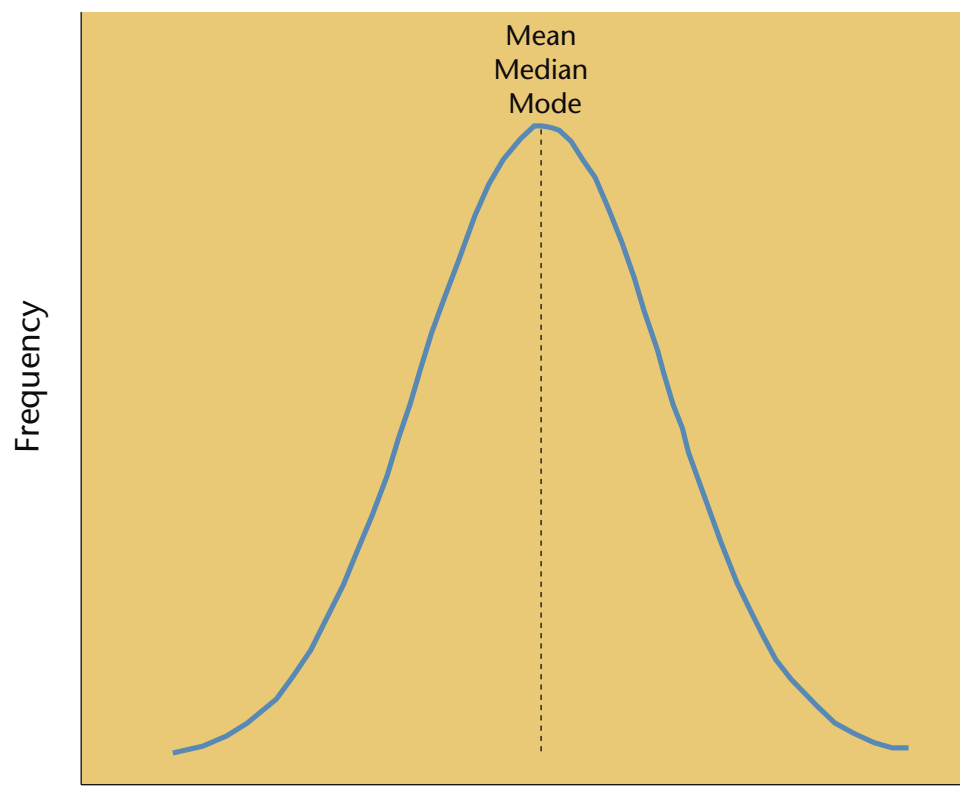

Normal curve with symmetrical distribution

Figure 1. The Normal (Gaussian)Distribution clinical benefit of an intervention. The null value for the ARR is 0 , and the null value for the NNT is infinity, meaning if the groups are identical, then an infinite number of patients would need to be treated to see any clinical benefit.

\section{Ordinal Data}

Before we discuss ordinal data statistics, a brief introduction to normal and skewed distributions is needed. If we took a large random sample of adult males living in London and wanted to know their height, it is likely this will follow a normal, or Gaussian distribution (Fig 1). With a normal distribution the mean (numerical average), median (middle value) and mode (most common value) are the same. Thus, the greatest number of men would be at the average height, with fewer men as we deviate from this average. Sometimes, data do not follow a normal distribution and can be skewed to the left or right as in Figure 2. Data such as the number of pregnancies are typically right-skewed, as there will be many women with either $0,1,2$, or 3 pregnancies but few with $4,5,6$, etc.

If our data set is $1,2,2,3,3,3,4,4,5$, this would be normally distributed with a mean, median, and mode taking a value of 3 . But changing just one value, say changing the " 5 " to " 50 " would make these data rightskewed. In this example, the mean is no longer 3, but the median value stays the same. In general, the median is much less sensitive to extreme values than is the mean.

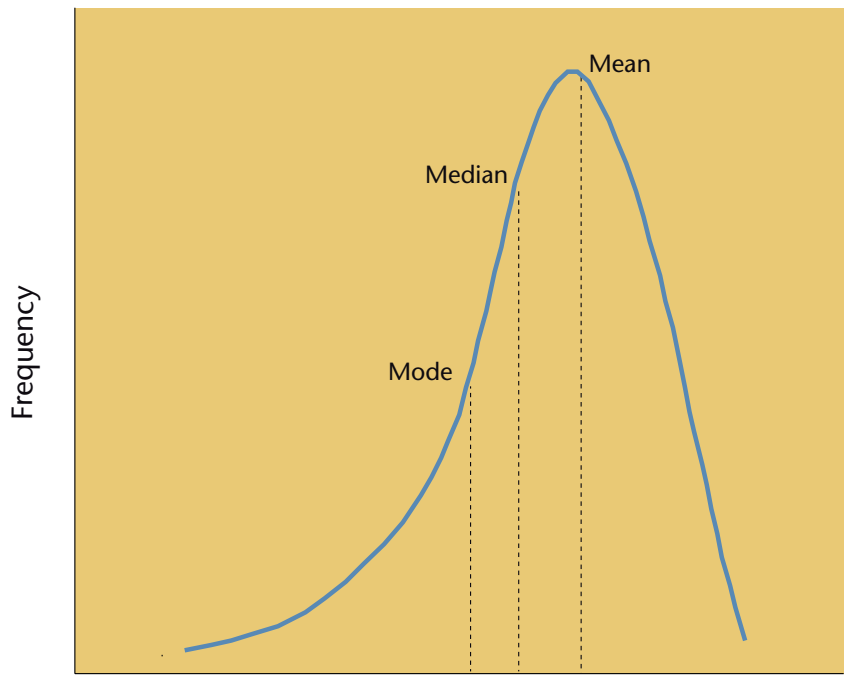

Negatively skewed curve

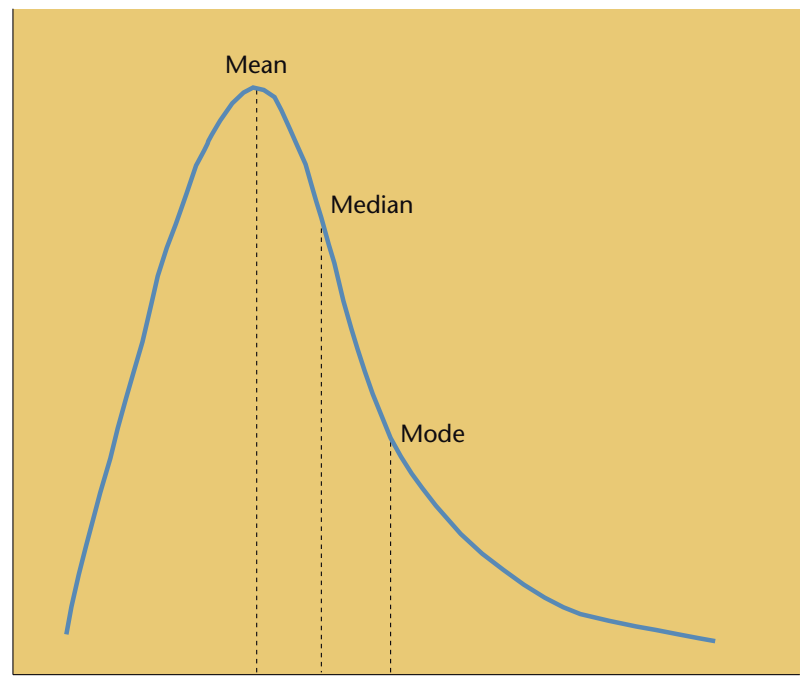

Positively skewed curve 


\begin{tabular}{|c|c|c|c|}
\hline & categorical & ordinal & quantitative \\
\hline description & $\begin{array}{l}\text { different categories of } \\
\text { data; none better than } \\
\text { the other }\end{array}$ & $\begin{array}{l}\text { different categories } \\
\text { with one level better } \\
\text { than the other, yet } \\
\text { imprecise }\end{array}$ & $\begin{array}{l}\text { real numbers with } \\
\text { precise meaning; } \\
\text { pocket depth of } 4 \mathrm{~mm} \\
\text { twice that of } 2 \mathrm{~mm}\end{array}$ \\
\hline examples & $\begin{array}{l}\text { eye colour, hair colour, } \\
\text { city of origin }\end{array}$ & $\begin{array}{l}\text { mild, moderate, or } \\
\text { severe periodontitis; } \\
\text { Likert scale }\end{array}$ & $\begin{array}{l}\text { age, height, alveolar } \\
\text { bone level }(\mathrm{mm})\end{array}$ \\
\hline common statistical test & chi-square & Mann-Whitney U & t-test \\
\hline how test works & $\begin{array}{l}\text { differences in } \\
\text { proportions }\end{array}$ & $\begin{array}{l}\text { differences in rank } \\
\text { order }\end{array}$ & differences in means \\
\hline \multirow[t]{2}{*}{ null value } & $x^{2}=0$ & median difference $=0$ & mean difference $=0$ \\
\hline & binary data & & \\
\hline description & only 2 categories & & \\
\hline examples & $\begin{array}{l}\text { male/female } \\
\text { disease/no disease }\end{array}$ & & \\
\hline common statistical test & $\begin{array}{l}\text { RR null value }=1 \\
\text { OR null value }=1\end{array}$ & & \\
\hline common statistical test & $\begin{array}{l}\text { ARR null value }=0 \\
\text { NNT null value }=\infty\end{array}$ & & \\
\hline
\end{tabular}

At this point we will introduce the terms parametric and non-parametric tests. Parametric refers to parameters of, in this case, the normal distribution, and parametric tests assume the data are normally distributed. Non-parametric tests make no such assumption on the distribution of the data, and generally look at differences in medians rather than means.

Let's look at a hypothetical parallel-group randomized trial of 10 patients comparing deep scaling alone to deep scaling and antibiotic gel. Our periodontal outcomes could be stages of periodontitis, expressed as none, mild, moderate or severe, which would be

outcome as periodontal pocket depth (quantitative data) for illustrative purposes. We are using a non-parametric test as the data are not normally distributed, often the case with small samples.

It appears that the scaling plus antibiotic gel group has done better than the control group. Statistical tests for skewed quantitative data, such as the Mann-Whitney U test, use the rank order of data values, rather than any values themselves. For example, if we had the following data sets for two groups of patients with different degrees of periodontitis, we would rank them in order of severity without using the actual data values. Because we are using rank rather than actual values, non-parametric tests such as the MannWhitney U test are not as precise, and thus, we need larger differences between groups to reach statistical significance.

\section{Quantitative Data}

Quantitative data represents numbers with precise mathematical meaning and statistical tests for this data type deal with differences in means. The most common hypothesis test for quantitative data is the t-test, which works by examining these differences. Let's examine our same randomized trial measuring differences in periodontal pocket depth. If the mean pocket depth in the control group was $6.0 \mathrm{~mm}$ and the mean depth in the test group was $5.0 \mathrm{~mm}$, the mean difference would be $6.0-5.0=1 \mathrm{~mm}$ (Table 3). Because we are dealing with actual numerical values, it is easier to achieve statistical significance with parametric tests such as the t-test compared with the non-parametric Mann-Whitney U. This is relevant as investigators occasionally use parametric tests with ordinal or skewed quantitative data.

\section{Summary} quantitative data deal with differences in proportions, rank order and means, respectively (Table 4). In the next paper, we will give hypothetical passages from dental studies using different data types, asking and answering a series of questions from these passages.

Evidence-Based Dentistry (2011) 12, 25-27. doi:10.1038/sj.ebd.6400781
Statistical tests for categorical, ordinal and 